Review

\title{
Agro-Nanotechnology as an Emerging Field: A Novel Sustainable Approach for Improving Plant Growth by Reducing Biotic Stress
}

\author{
Masudulla Khan ${ }^{1, *(1)}$, Azhar U. Khan ${ }^{2, *}$, Mohd Abul Hasan ${ }^{3}\left(\mathbb{D}\right.$, Krishna Kumar Yadav ${ }^{4}\left(\mathbb{D}\right.$, Marina M. C. Pinto ${ }^{5, *}$, \\ Nazia Malik ${ }^{6}$, Virendra Kumar Yadav ${ }^{7, *}$, Afzal Husain Khan ${ }^{8}{ }^{10}$, Saiful Islam ${ }^{3}$ and Gulshan Kumar Sharma ${ }^{9}$ \\ Citation: Khan, M.; Khan, A.U.; \\ Hasan, M.A.; Yadav, K.K.; Pinto, \\ M.M.C.; Malik, N.; Yadav, V.K.; Khan, \\ Department of Botany, Aligarh Muslim University, Aligarh U.P. 202002, India \\ 2 School of Life and Basic Sciences, Department of Chemistry, SIILAS CAMPUS, Jaipur National University, \\ Jaipur 302017, India \\ 3 Civil Engineering Department, College of Engineering, King Khalid University, Abha 62529, Saudi Arabia; \\ mohad@kku.edu.sa (M.A.H.); sfakrul@kku.edu.sa (S.I.) \\ 4 Institute of Environment and Development Studies, Bundelkhand University, Kanpur Road, \\ Jhansi 284128, India; envirokrishna@gmail.com \\ 5 Geobiotec Research Centre, Department of Geoscience, University of Aveiro, 3810-193 Aveiro, Portugal \\ 6 Department of Chemistry, Aligarh Muslim University, Aligarh U.P. 202002, India; \\ nazia.amu123@rediffmail.com \\ 7 School of Lifesciences, SIILAS CAMPUS, Jaipur National University, Jaipur 302017, India \\ 8 Civil Engineering Department, College of Engineering, Jazan University, Jazan 114, Saudi Arabia; \\ ahkhan@jazanu.edu.sa \\ 9 National Bureau of Soil Survey and Land Use Planning, Regional Centre Jorhat, Assam 785001, India; \\ gulshansharma2222@gmail.com \\ * Correspondence: masudkhann@gmail.com (M.K.); azhar.u.kh@gmail.com (A.U.K.); \\ marinacp@ua.pt (M.M.C.P.); virendra.yadav@jnujaipur.ac.in (V.K.Y.)
} A.H.; Islam, S.; Sharma, G.K. Agro-Nanotechnology as an Emerging Field: A Novel Sustainable Approach for Improving Plant Growth by Reducing Biotic Stress. Appl. Sci. 2021, 11, 2282. https:// doi.org/10.3390/app11052282

Academic Editor: Anthony William Coleman

Received: 27 January 2021

Accepted: 23 February 2021

Published: 4 March 2021

Publisher's Note: MDPI stays neutral with regard to jurisdictional claims in published maps and institutional affiliations.

Copyright: (c) 2021 by the authors. Licensee MDPI, Basel, Switzerland. This article is an open access article distributed under the terms and conditions of the Creative Commons Attribution (CC BY) license (https:// creativecommons.org/licenses/by/ $4.0 /)$.
Abstract: In the present era, the global need for food is increasing rapidly; nanomaterials are a useful tool for improving crop production and yield. The application of nanomaterials can improve plant growth parameters. Biotic stress is induced by many microbes in crops and causes disease and high yield loss. Every year, approximately $20-40 \%$ of crop yield is lost due to plant diseases caused by various pests and pathogens. Current plant disease or biotic stress management mainly relies on toxic fungicides and pesticides that are potentially harmful to the environment. Nanotechnology emerged as an alternative for the sustainable and eco-friendly management of biotic stress induced by pests and pathogens on crops. In this review article, we assess the role and impact of different nanoparticles in plant disease management, and this review explores the direction in which nanoparticles can be utilized for improving plant growth and crop yield.

Keywords: plant diseases; nanoparticles; diseases; biotic stress; management; silver nanoparticles; zinc nanoparticles

\section{Introduction}

Crop cultivators suffer from high yield loss caused by various diseases. Biotic stress induced by microbes on crop plants reduces the crop yield and decreases the quality. Biotic stress causes disease in crops, which leads to the suffering of the plant. Diseases of the plant need to be controlled to maintain the abundance of food produced by farmers around the world. The management of crop diseases is very necessary to fulfill the food demand. Potato blight disease caused by plant pathogenic fungus Phytopthora caused more than one million deaths in Ireland [1]. Around $20-40 \%$ of agricultural crop yield losses occur globally due to various diseases caused by phytopathogenic bacteria, phytopathogenic fungi, pests, and weeds [2]. 
It is estimated that in 2050 the world's human population will reach around 10 billion, and around 800 million people in the world will be hungry and around 653 million people in the world will be undernourished in 2030, thus fulfilling the food demand will remain a huge challenge. The current research progress and disease management strategies are not enough to fulfill the food demand by 2050 [3]. The first green revolution made a huge difference in yield and food production, but in the last few years' crop production has been stagnant and food demand is increasing sharply, so now we need a second green revolution to fulfill the food demand of the population.

Different approaches are used by farmers to mitigate the impact of plant diseases. The agriculture system mainly relies on chemicals to manage crop diseases and inhibit the growth of phytopathogens, which cause diseases before and after crop harvesting. The excessive use of chemical pesticides, herbicides, and fungicides that are mainly used to control plant diseases causes harmful environmental and human health consequences. Tilman et al. [4] observed that the high use of chemical pesticides increases resistance in pathogens and pests, reduces nitrogen fixation, and the bioaccumulation of toxic pesticides occurs.

An example is the synthetic chemical pesticide DDT, dichlorodiphenyltrichloroethane, which was extensively used in agriculture for controlling plant pathogens and was found to be genotoxic in humans, causing endocrine disorders [5]. Water and soil pollution is also caused by the excessive use and misuse of these chemicals. There is an increasing demand day by day to reduce the use of synthetic chemicals. Consequently, the harmful effects of chemicals on wildlife, the environment, and human health have increased the need for alternative measures in the control of plant pathogens, so that some phytopathologists have focused their research on developing a new alternative that should replace the use of chemicals in controlling plant diseases.

Nanotechnology has revolutionized agriculture and can control plant diseases, although the field of nanotechnology is still in the nascent stage and needs more research analysis [6].The use of nanomaterials in agriculture will reduce the excessive use of toxic chemicals used for plant disease management (Figures 1 and 2).

"Nano" denotes one-billionth part, thus nanotechnology deals with small things. The word nano is used for materials with a size range of 0.1 to 100 nanometers $[7,8]$. The first time the term nanotechnology was used was by Taniguchi in 1974 to the science that largely deals with particles of nano size $\left(1.0 \times 10^{-9} \mathrm{~m}\right)$. When a bulk material is reduced to nano size, it has a high surface-to-volume ratio that may increase its reactivity and express some new properties [7,9]. The control of plant diseases and improving plant growth by the use of nanomaterials are some of the possible key applications in the area of plant pathology. Approximately 260,000-309,000 metric tons of nanoparticles were produced in 2010 globally, and the worldwide consumption of nanomaterials was approximately from 225,060 metric tons to 585,000 metric tons in 2014 to 2019 [10,11].

In this review article, recent research progress and the application of various nanoparticles for the sustainable management of the biotic stress of crop systems and impact on plant growth have been discussed. We try to cover the various problems associated with crop cultivation and plant diseases and the use of different nanomaterials to control phytopathogens and improve plant growth. 


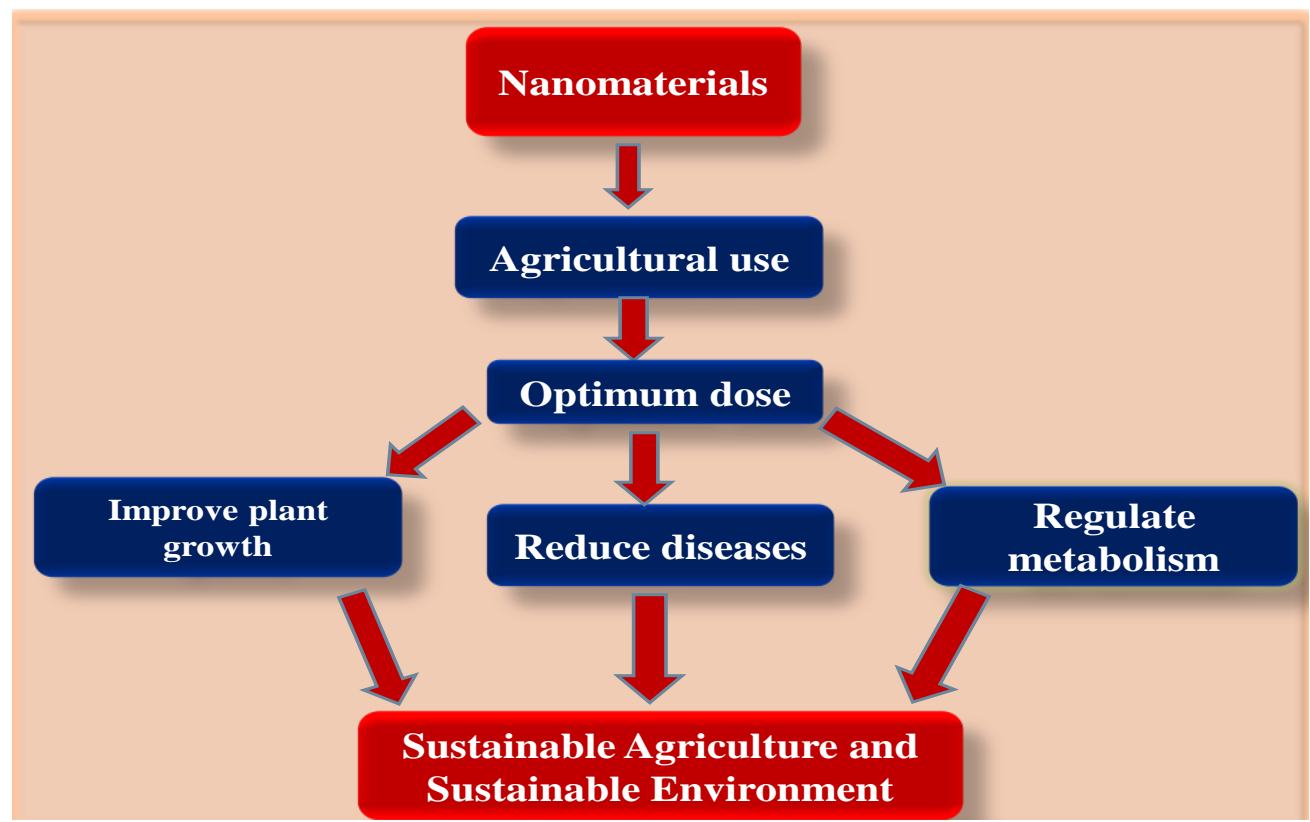

Figure 1. Schematic presentation of nanomaterials in agriculture [12].

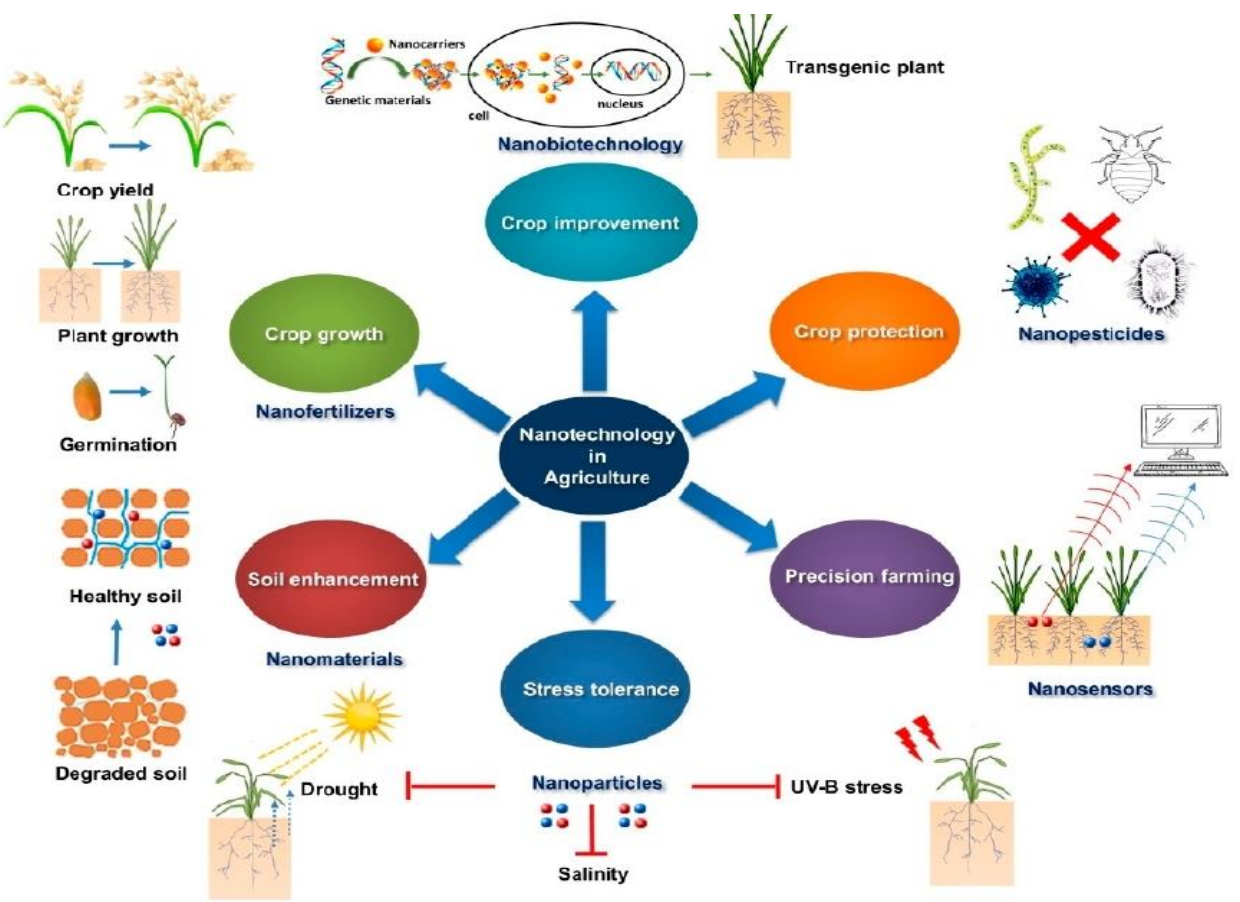

Figure 2. Various applications of nanotechnology in agriculture taken from [12].

\section{Nanomaterials in Improving Plant Growth and Yield}

Currently, around 1300 nanomaterials, with widespread potential applications, are available $[13,14]$. Nanoparticles can penetrate the cell wall because the cell wall is porous to $3.5-20 \mathrm{~nm}$ macromolecules. Nanoparticles can enter through stomatal openings. When stomata are present at the lower surface of leaves, the entry of nanoparticles (NPs) becomes difficult [15]. It is reported that nanoparticles of size $\leq 43 \mathrm{~nm}$ can penetrate and enter into stomata $[16,17]$.

The effect of nanoparticles on crop plants is concentration-based. Many plant processes such as seed germination and plant growth are affected by NP concentration [18]. Many NPs have been reported to be beneficial for plant growth. Mahmoud et al. [19] used 
$\mathrm{Zn}, \mathrm{B}, \mathrm{Si}$, zeolite NPs on a potato plant and found that these nanoparticles have a positive effect on potato plants and they improve the plant growth. Khan and Siddiqui [20] treated eggplant with ZnONPs and found a foliar spray of ZnONPs causes the highest improvement in eggplant growth. Awasthi et al. [21] reported that ZnONPs have a positive effect on seed germination in the Triticum aestivum plant. Zinc oxide nanoparticles (ZnONPs) can enhance plant biomass and agriculture production [22]. Sabir et al. [23] also showed that nanocalcite $\left(\mathrm{CaCO}_{3}\right)$ application with $\mathrm{Fe}_{2} \mathrm{O}_{3}$, nano $\mathrm{SiO}_{2}$, and $\mathrm{MgO}$ improved the uptake of $\mathrm{Mg}, \mathrm{Ca}$, and $\mathrm{Fe}$, and also notably enhanced the intake of $\mathrm{P}$ with micronutrients $\mathrm{Zn}$ and Mn. Venkatachalam et al. [24] found that ZnONPs increase in photosynthetic pigment in the Leucaena leucocephala plant. Narendhran et al. [25] reported high chlorophyll-a', chlorophyll-' $\mathrm{b}$ ' and total chlorophyll content in the Sesamum indicum plant when treated with ZnO NPs. Taheri et al. [26] observed that treatment of ZnONPs increases the increase in shoot dry matter in Zea mays. Tarafdar et al. [27] found that ZnONPs enhanced shoot and grain yield in the Pennisetum glaucum plant.

The application of titanium dioxide $\left(\mathrm{TiO}_{2}\right)$ on crops promotes plant growth parameters and can enhance the photosynthetic rate. Siddiqui et al. [28] usedTiO ${ }_{2}$ and $\mathrm{ZnONPs} \mathrm{on}^{2}$ beet root plants. They found that both NPs increased chlorophyll and carotenoid content, improved plant growth, and also improved super oxide dismutase (SOD), catalase (CAT), $\mathrm{H}_{2} \mathrm{O}_{2}$, and proline content in plants. ZnONPs were found to be better than $\mathrm{TiO}_{2} \mathrm{NPs}$ on beetroot plants. Raliya et al. [29] reported that $\mathrm{TiO}_{2} \mathrm{NPs}_{\text {s }}$ treatment improved shoots in the Vigna radiate plant. Lawre and Raskar [30] observed that $\mathrm{TiO}_{2} \mathrm{NPs}$ at a lower concentration enhanced seed germination and seedling growth in onion plants. Rafique et al. [31] found a positive effect of $\mathrm{TiO}_{2} \mathrm{NPs}$ on the Triticum aestivum plant. Mahmoodzadeh et al. [32] found a positive effect of $\mathrm{TiO}_{2} \mathrm{NPs}$ on the seed germination of the Brassica napus plant. Qi et al. [33] reported that treatment of $\mathrm{TiO}_{2} \mathrm{NPs}$ promotes photosynthetic rate in tomato plants.

Silicon is an important element that plays a key role in several metabolic and physiological activities in plants [34]. $\mathrm{SiO}_{2}$ nanoparticles have the potential to enhance the germination and seedling growth of Agropyron elongatum [35]. $\mathrm{Nano}^{-\mathrm{SiO}_{2}}$ can be used to produce effective fertilizers for crops and to minimize the loss of fertilizer through slow and controlled release, allowing for regulated, responsive, and timely delivery [36]. Siddiqui et al. [37] found improved seed germination in the Cucurbita pepo plant after treatment with $\mathrm{Nano} \mathrm{SiO}_{2}$. Haghighi and Pessarakli [38] reported that Nano Si treatment on the tomato plant improves photosynthetic rate in treated plants.

Copper is an essential element for plant growth and development. Copper plays a key role in the activity of many plant enzymes. Copper nanoparticles (Cu NP) are used as antimicrobial agents, gas sensors, catalysts, electronics, etc. [39]. Wang et al. [40] found that $\mathrm{CuO}$ NPs improved photosynthesis in the Spinacia oleracea plant. Zhao et al. [41] reported that $\mathrm{Cu}(\mathrm{OH})_{2} \mathrm{NPs}$ improved the antioxidant system of the Lactuca sativa plant. Shinde et al. [42] found that $\mathrm{Mg}(\mathrm{OH})_{2} \mathrm{NP}$ treatment promotes seed germination and seedling growth in the Zea mays plant. Hussain et al. [43] reported that MgO NPs improve the antioxidant system in Raphanus sativus plants. Cai et al. [44] observed that $\mathrm{MgO}$ NPs can promote the plant growth of the Tobacco plant. Imada et al. [45] found that MgO NPs can induce resistance in the tomato plant.

Iqbal et al. [46] reported that AgNP treatment improved plant growth and tolerance to heat stress in the Triticum aestivum plant. Mehta et al. [47] found that AgNPs' foliar application enhanced growth and biomass in the Vigna sinensis plant. Pilon et al. [48] observed that chitosan NPs protect apple plants after post-harvest. Van et al. [49] found that chitosan NPs improve plant growth in Robusta coffee.

Das et al. [50] found that $\mathrm{FeS}_{2}$ NPs improved seed germination in Cicer arietinum, Daucus carota, pinacia oleracea, Brassica juncea, and Sesamum indicum crops. The effects of various nanomaterials have been summarized in the following table (Table 1). 
Table 1. Effect of various nanomaterials on plant physiology and growth parameters.

\begin{tabular}{|c|c|c|c|}
\hline Nanoparticles & Plant & Effect on Plants in a Dose-Dependent Manner & Reference \\
\hline Zn, B, Si, Zeolite NPs & Potato & Improve plant growth & [19] \\
\hline ZnO NPs & Eggplant & Increase plant growth attributes & [20] \\
\hline $\mathrm{ZnO}$ NPs & Triticum aestivum & Positive effect on seed germination & [21] \\
\hline $\mathrm{SiO}_{2} \& \mathrm{TiO}_{2} \mathrm{NPs}$ & Rice & Improve plant growth attributes & [22] \\
\hline $\begin{array}{c}\text { Nano-size calcite product } \\
\text { [CaCO3(40\%), } \mathrm{SiO} 2(4 \%), \mathrm{MgO} \\
(1 \%), \text { and } \mathrm{Fe} 2 \mathrm{O} 3(1 \%)]\end{array}$ & Grapevine & $\begin{array}{l}\text { Increase plant growth attributes and } \\
\text { photosynthetic pigment }\end{array}$ & [23] \\
\hline $\mathrm{ZnO} \mathrm{NPs}$ & Leucaena leucocephala & $\begin{array}{l}\text { Increase in photosynthetic pigment and total } \\
\text { soluble protein contents }\end{array}$ & [24] \\
\hline $\mathrm{ZnO}$ NPs & Sesamum indicum & $\begin{array}{l}\text { High chlorophyll'a', chlorophyll'b', and total } \\
\text { chlorophyll content level }\end{array}$ & [25] \\
\hline ZnO NPs & Zea mays & Increased shoot dry matter and leaf area indexes. & [26] \\
\hline ZnO NPs & Pennisetum glaucum & ZnO NPs enhanced shoot and grain yield & [27] \\
\hline $\mathrm{TiO}_{2} \& \mathrm{ZnO} \mathrm{NPs}$ & Beetroot & Increased plant growth and shoot dry matter & [28] \\
\hline $\mathrm{TiO}_{2} \mathrm{NPs}$ & Vigna radiata $\mathrm{L}$. & Improvement was observed in shoot length & [29] \\
\hline $\mathrm{TiO}_{2} \mathrm{NPs}$ & Onion & $\begin{array}{l}\text { Lower concentration of } \mathrm{TiO}_{2} \mathrm{NPs} \text { enhanced seed } \\
\text { germination and seedlings growth }\end{array}$ & [30] \\
\hline $\mathrm{TiO}_{2} \mathrm{NPs}$ & Triticum aestivum $\mathrm{L}$. & Increase in the plant's root and shoot lengths & [31] \\
\hline $\mathrm{TiO}_{2}$ NPs & Brassica napus & $\begin{array}{c}\text { Promoted seed germination and seedling } \\
\text { vigor improved }\end{array}$ & [32] \\
\hline $\mathrm{TiO}_{2} \mathrm{NPs}$ & Tomato & Promote the photosynthetic rate & {$[33]$} \\
\hline $\mathrm{SiO}_{2} \mathrm{NPs}$ & Larix olgensis & $\begin{array}{l}\text { Increase in plant height, root length, } \\
\text { and chlorophyll content }\end{array}$ & [34] \\
\hline $\mathrm{SiO}_{2} \mathrm{NPs}$ & Agropyron elongatum L. & Improve seed germination & [35] \\
\hline Nano- $\mathrm{SiO}_{2}$ & Cucurbita pepo L. & Reduce the salt stress effect & [37] \\
\hline Nano Si & Tomato & Enhancement of germination rate and dry weight & [38] \\
\hline $\mathrm{CuO}$ NPs & Spinacia oleracea & Improved photosynthesis in treated plants & [40] \\
\hline $\mathrm{MgO}$ NPs & Tobacco & Promote plant growth & [44] \\
\hline $\mathrm{MgO}$ NPs & Tomato & Induce resistance in tomato plant & [45] \\
\hline AgNPs & Wheat & Regulate antioxidative defence system & [46] \\
\hline AgNPs & soil bacterial diversity & Regulate soil bacterial diversity & [47] \\
\hline Chitosan NPs & Apples & They reduce microbial growth & [48] \\
\hline Chitosan NPs & Robusta cofee & Improved growth parameters & [49] \\
\hline $\mathrm{FeS}_{2} \mathrm{NPs}$ & $\begin{array}{l}\text { Cicer arietinum; pinacia oleracea; } \\
\text { Daucus carota, Brassica juncea } \\
\text { and Sesamum indicum }\end{array}$ & Seed germination enhanced in tested crops & {$[50]$} \\
\hline Chitosan NPs & Rice & Reduces disease severity & [51] \\
\hline Chitosan NPs & Strawberry & Regulate defense response & [52] \\
\hline SiNPs & Helianthus annuus & Improved germination & [53] \\
\hline SilicaNPs & Vicia faba L. & Improved growth parameters & [54] \\
\hline $\mathrm{SiO}_{2} \mathrm{NPs}$ & Pea & $\begin{array}{l}\text { Improved growth parameters and } \\
\text { chlorophyll content }\end{array}$ & [55] \\
\hline $\mathrm{SiO}_{2} \& \mathrm{MoNPs}$ & Rice & Regulate seed germination & [56] \\
\hline $\mathrm{SiO}_{2} \mathrm{NPs}$ & Indocalamus barbatus & Improved photosynthetic pigments & [57] \\
\hline
\end{tabular}


Table 1. Cont.

\begin{tabular}{|c|c|c|c|}
\hline Nanoparticles & Plant & Effect on Plants in a Dose-Dependent Manner & Reference \\
\hline SilicaNPs & Zea mays. L & Improve silica content in plants & [58] \\
\hline $\mathrm{SiO}_{2} \mathrm{NPs}$ & Maize & $\begin{array}{l}\text { Improved growth parameters and increased } \\
\text { seed stability }\end{array}$ & [59] \\
\hline $\mathrm{SiO}_{2}$ and $\mathrm{TiO}_{2} \mathrm{NPs}$ & Soybean & Enhance germination of seeds & [60] \\
\hline $\mathrm{Cu}(\mathrm{OH})_{2}$ & Lactuca sativa & Improve antioxidant system & [61] \\
\hline $\mathrm{Cu}(\mathrm{OH})_{2}$ & Spinach & Improve the antioxidant system & {$[62]$} \\
\hline ZnO NPs & Glycine max & Enhanced Antioxidant system & [63] \\
\hline $\mathrm{ZnO}$ NPs & $\begin{array}{l}\text { Cabbage, cauliflower, } \\
\text { and tomato }\end{array}$ & Enhance pigments, protein, and sugar contents & {$[64]$} \\
\hline ZnO NPs & Arachis hypogaea & Seed germination enhaced & [65] \\
\hline $\mathrm{FeS}_{2} \mathrm{NP}$ & Spinach & Improve plant growth & {$[66]$} \\
\hline $\mathrm{TiO}_{2} \mathrm{NPs}$ & Glycine max L. & $\begin{array}{l}\text { Positive effect on the seed and oil yield and } \\
\text { component compared to the control }\end{array}$ & [67] \\
\hline $\mathrm{TiO}_{2} \mathrm{NPs}$ & Mentha Piperita & Increased root length & [68] \\
\hline $\mathrm{TiO}_{2} \mathrm{NPs}$ & Agropyron desertorum & Improves seed germination & [69] \\
\hline
\end{tabular}

\section{Nanomaterials in Various Diseases Management}

Nanomaterials have antimicrobial activity. Silver nanoparticles have anti-bacterial and anti-fungal properties. Kim et al. [70] have reported the fungicidal effects of nanosilver against Alternaria alternata, A. brassicicola, A. solani, Botrytis cinerea, Cladosporium cucumerinum, Corynespora cassiicola, Cylindrocarpon destructans, Didymella bryoniae, Fusarium oxysporum f. sp. cucumerinum, F. oxysporum f. sp. lycopersici, F. oxysporum, F. solani, Fusarium sp., Glomerella cingulata and a few other fungi. Gautam et al. [71] showed the antifungal and antibacterial activity of AgNPs against Erwinia sp., Bacillus megaterium, Pseudomonas syringe, Fusarium graminearum, F. avenaceum, and F. culmorum fungi. Rodríguez-Serrano et al. [72] reported the antibacterial activity of AgNPs against E. coli. Husseinet al. [73] reported the antibacterial activity of AgNPs against Staphylococcus aureus and Klebsiella pneumonia. Shehzad et al. [74] reported that AgNPs have antibacterial activity against Gram-positive (Bacillus subtilis) and Gram-negative (Escherichia coli) bacteria. Mohanta et al. [75] reported that AgNPs have antibacterial activity against food borne pathogens Pseudomonas aeruginosa, Escherichia coli, and Bacillus subtilis. Abdelmale and Salaheldin [76] reported that AgNPs show antifungal activity against Alternaria alternata, A. citri, and Penicillium digitatum fungi. Krishnaraj et al. [77] found theantifungal activity of AgNPs against Alternaria alternata, Macrophomina phaseolina, Botrytis cinerea, Sclerotinia sclerotiorum, Curvularia lunata, and Rhizoctonia solani fungi. Jo et al. [78] described the antifungal activity of AgNPs against Bipolaris sorokiniana and Magnaporthe grisea fungi.

Shahryari et al. [79] reported that AgNPs and a silver-chitoson composite show antibacterial activity against Pseudomonas syringae pv. syringae bacteria. Divya et al. [80] reported that chitoson NPs have antifungal activity against Macrophomia phaseolina and Alternaria alterneta fungi. Xing et al. [81] reported that chitoson NPs have antifungal activity against Fusarium solani and Aspergillus niger fungi. Dang et al. [82] reported that AuNPs have antibacterial activity against E. coli bacteria. Attar and Yapaoz [83] observed that $\mathrm{ZnO}$ and AuNPs have antibacterial activity against $E$. coli bacteria. The gold nanoparticles showed toxic effect on bacteria, Salmonella typhimurium, in which the macro gold did not exhibit. Jayaseelana et al. [84] synthesized gold nanoparticles from Abelmoschus esculentus and reported their antifungal activity. The antifungal activity of AuNPs was tested against Puccinia graministritci, Aspergillus niger, Aspergillus flavus and Candida albicans using the standard well diffusion method. The maximum zone of inhibition was observed in the Au NPs against $P$. graminis and $C$. albicans. 
Fan et al. [85] observed the antibacterial activity of $\mathrm{Cu}$ composites against Xanthomonas euvesicatoria. Huang et al. [86] showed the antifungal activity of CuO NPs against Botrytis cinerea, Colletotrichum graminicola, Rhizoctonia solani, Colletotrichum musae, Magnaporthe oryzae, Penicillium digitatum, and Sclerotium rolfsii. Giannousiet al. [87] showed the antifungal activity of $\mathrm{CuO}$ and $\mathrm{Cu}_{2} \mathrm{O}$ NPs against Phytophthora infestans. Sharmaet al. [88] reported the antifungal and antibacterial activity of MgONPs against Ralstonia solanacearum bacteria and Phomopsis vexans fungus. Imada et al. [45] found the antibacterial activity of MgONPs against Ralstonia solanacearum. Derbalah et al. [89] observed the antifungal property of silica NPs against Alternaria solani fungus. Akpinar et al. [90] found that $\mathrm{SiO}_{2}$ NPs possess antifungal properties against Fusarium oxysporum $\mathrm{f}$. sp. lycopersici and $F$. oxysporum f. sp. radicislycopersici. Park et al. [91] showed the antifungal activity of Nano Si-Ag against Pythium ultimum, Magnaporthe grisea, Colletotrichum gloeosporioides, Botrytis cineria, Rhizoctonia solani, Pseudomonas syringae, Xanthomonas compestris pv. vesicatoria.

Jamdagni et al. [92] found that ZnO NPs have promising antifungal activity against Alternaria alternate Botrytis cinerea, Aspergillus niger, Fusarium oxysporum, and Penicillium expansum fungi. Navale et al. [93] found the promising antifungal activity of ZnO NPs against Aspergillus flavus and Aspergillus fumigates fungi. Rajiv et al. [94] reported the antifungal activity of $\mathrm{ZnO}$ NPs against Aspergillus flavus, A. niger, A. fumigates, Fusarium culmorum, and F. oxysporium. Gunalan et al. [95] found that $\mathrm{ZnO}$ NPs have promising antifungal activity against Aspergillus flavus, Trichoderma harzianum, A. nidulans, and Rhizopus stolonifer. Dimkpa et al. [96] have shown the antifungal activity of $\mathrm{ZnO}$ nanoparticles on Fusarium graminearum fungus. Jayaseelan et al. [97] synthesized $\mathrm{ZnO}$ nanoparticles using Aeromonas hydrophila and screened their activity against pathogenic bacteria $P$. aeruginosa, and fungi, C. albicans, A. flavus, and A. niger. Sar et al. [98] reported the antifungal activity of $\mathrm{TiO}_{2}$ NPs against Fusarium oxysporum f. sp. radicislycopersici and Fusarium oxysporum f. sp. Lycopersici. Hamza et al. [99] found the antifungal activity of $\mathrm{TiO}_{2} \mathrm{NPs}$ against Cercospora beticola. Ardakani [100] found the nematicidal activity of $\mathrm{TiO}_{2} \mathrm{NPs}_{\text {against Meloidogyne }}$ incognita nematode. Kasemets et al. [101] reported the antifungal activity of $\mathrm{ZnO}$ and $\mathrm{TiO}_{2}$ NPs against Saccharomyces cerevisiae. Cui et al. [102] found that $\mathrm{TiO}_{2} \mathrm{NPs}$ have antibacterial against P. syringae pv. lachrymans and P. cubensis (Table 2, Figure 3).

Table 2. Various nanomaterials in plant disease management

\begin{tabular}{|c|c|c|c|}
\hline Nanoparticle & Pathogen & Effect & Reference \\
\hline Ag NPs & $\begin{array}{c}\text { Alternaria alternata, A. brassicicola, A. solani, } \\
\text { Cladosporium cucumerinum, Botrytis cinerea, } \\
\text { Corynespora cassiicola, Cylindrocarpon destructans, } \\
\text { Didymella bryoniae, F. oxysporum f. sp. lycopersici, F. } \\
\text { oxysporum, Fusarium oxysporum f.sp. cucumerinum, F. } \\
\text { solani, Fusarium sp., Glomerella cingulata, P. spinosum, } \\
\text { Monosporascuscannonballus, Pythium aphanidermatum, } \\
\text { Stemphylium lycopersici }\end{array}$ & Show antifungal activity & {$[70]$} \\
\hline AgNPs & $\begin{array}{l}\text { Erwinia sp., Bacillus megaterium, Pseudomonas syringe, } \\
\text { Fusarium graminearum, F. avenaceum, F. culmorum }\end{array}$ & $\begin{array}{l}\text { An inhibitory effect on } \\
\text { tested microbes }\end{array}$ & {$[71]$} \\
\hline AgNPs & Escherichia coli & Antibacterial activity & [72] \\
\hline AgNPs & Staphylococcus aureus and Klebsiella pneumonia & Antibacterial activity & [73] \\
\hline AgNPs & $\begin{array}{l}\text { Gram-positive (Bacillus subtilis) and gram-negative } \\
\text { (Escherichia coli). }\end{array}$ & $\begin{array}{l}\text { An inhibitory effect on } \\
\text { tested bacteria }\end{array}$ & [74] \\
\hline AgNPs & $\begin{array}{l}\text { Foodborne pathogens viz. Pseudomonas aeruginosa, } \\
\text { Escherichia coli, Bacillus subtilis. }\end{array}$ & Antibacterial activity & [75] \\
\hline AgNPs & Alternaria alternata, A. citri, Penicillium digitatum & Show antifungal properties & [76] \\
\hline AgNPs & $\begin{array}{c}\text { Alternaria alternata, Macrophomina phaseolina, Botrytis } \\
\text { cinerea, Sclerotinia Sclerotiorum, Curvularia lunata, } \\
\text { Rhizoctonia solani }\end{array}$ & Show Antifungal activity. & [77] \\
\hline
\end{tabular}


Table 2. Cont.

\begin{tabular}{|c|c|c|c|}
\hline Nanoparticle & Pathogen & Effect & Reference \\
\hline AgNPs & Bipolaris sorokiniana and MagnaportheGrisea & Show antifungal activity & [78] \\
\hline $\begin{array}{l}\text { AgNPs and Cs-Ag } \\
\text { nanocomposite }\end{array}$ & Pseudomonas syringaepv.syringae & Show antibacterial activity & [79] \\
\hline Chitosan NPs & $\begin{array}{c}\text { Klebsiella pneumoniae, Escherichia coli, Staphylococcus } \\
\text { aureus, Pseudomonas aeruginosa }\end{array}$ & Show antibacterial activity & [80] \\
\hline Chitosan NPs & Fusarium solani, Aspergillus niger & Show Antifungal activity & [81] \\
\hline $\mathrm{Au}$ NPs & Escherichia coli and Staphylococcus & Antibacterial activity & [82] \\
\hline $\mathrm{ZnO}$ and $\mathrm{Au} \mathrm{NPs}$ & E. coli & Antibacterial activity & [83] \\
\hline AuNPs & $\begin{array}{c}\text { Puccinia graminis tritci, Aspergillus flavus, Aspergillus } \\
\text { niger and Candida albicans }\end{array}$ & Show Antifungal activity & [84] \\
\hline Cu composites & Xanthomonas euvesicatoria & Antibacterial activity & [85] \\
\hline CuO NPs & $\begin{array}{c}\text { Botrytis cinerea, Colletotrichumgraminicola, Rhizoctonia } \\
\text { solani, Colletotrichum musae, Magnaportheoryzae, } \\
\text { Penicillium digitatum, Sclerotium rolfsii }\end{array}$ & Show antifungal activity & [86] \\
\hline $\mathrm{CuO}$ and $\mathrm{Cu}_{2} \mathrm{O}$ NPs & Phytophthora infestans & Show antifungal activity & [87] \\
\hline $\mathrm{MgO}$ NPs & Ralstonia solanacearum, Phomopsis vexans & $\begin{array}{l}\text { Show antifungal and } \\
\text { antibacterial activity }\end{array}$ & [88] \\
\hline SilicaNPs & Alternaria $s p$ & Show antifungal activity & [89] \\
\hline $\mathrm{SiO}_{2} \mathrm{NPs}$ & $\begin{array}{l}\text { Fusarium oxysporum f. sp. lycopersici and } F \text {. } \\
\text { oxysporum f. sp. radicislycopersici }\end{array}$ & Possess antifungal properties & [90] \\
\hline Nano Si-Ag & $\begin{array}{c}\text { Pythium ultimum, Magnaporthe grisea, Colletotrichum } \\
\text { gloeosporioides, Botrytis cineria, Rhizoctonia solani, } \\
\text { Pseudomonas syringae, Xanthomonas compestris } \\
\text { pv. vesicatoria }\end{array}$ & $\begin{array}{l}\text { Show antifungal and } \\
\text { antibacterial activity }\end{array}$ & [91] \\
\hline $\mathrm{ZnO}$ NPs & $\begin{array}{l}\text { Alternaria alternate Botrytis cinerea, Aspergillus niger, } \\
\text { Fusarium oxysporum and Penicillium expansum }\end{array}$ & $\begin{array}{l}\text { Antifungal activity against all } \\
\text { the tested fungi }\end{array}$ & [92] \\
\hline $\mathrm{ZnO}$ NPs & Aspergillus flavus and Aspergillus fumigates & $\begin{array}{l}\text { Shown potential activity } \\
\text { against these tested fungi }\end{array}$ & [93] \\
\hline $\mathrm{ZnO}$ NPs & $\begin{array}{l}\text { Aspergillus flavus, A. niger, A. fumigatus } \\
\text { Fusarium culmorum and F. oxysporium }\end{array}$ & $\begin{array}{l}\text { The highest zone of inhibition } \\
\text { occurred in } A \text {. flavus }\end{array}$ & [94] \\
\hline $\mathrm{ZnO}$ NPs & $\begin{array}{c}\text { Aspergillus flavus, A. nidulans, Trichoderma harzianum } \\
\text { and Rhizopus stolonifer }\end{array}$ & Antifungal activity & [95] \\
\hline ZnO NPs & Fusarium graminearum & Antifungal activity & [96] \\
\hline $\mathrm{ZnO} \mathrm{NPs}$ & Pseudomonas aeruginosa & Antibacterial activity & [97] \\
\hline $\mathrm{TiO}_{2}$ NPs & $\begin{array}{l}\text { Fusarium oxysporum f. sp. radicislycopersici and } \\
\text { Fusarium oxysporum f. sp. Lycopersici }\end{array}$ & Antifungal activity & [98] \\
\hline $\mathrm{TiO}_{2} \mathrm{NPs}$ & Cercosporabeticola & $\begin{array}{l}\text { Pathogen growth } \\
\text { was inhibited }\end{array}$ & [99] \\
\hline $\mathrm{TiO}_{2} \mathrm{NPs}$ & Meloidogyne incognita & Controlled M. incognita & {$[100]$} \\
\hline $\begin{array}{l}\mathrm{TiO}_{2} \text { NPs and } \mathrm{ZnO} \\
\text { NPs }\end{array}$ & Saccharomyces cerevisiae & Antifungal activity & [101] \\
\hline $\mathrm{TiO}_{2} \mathrm{NPs}$ & P. syringaepv. lachrymans and P. cubensis & Reduced infection of pathogen & {$[102]$} \\
\hline Metallic NPs & Fungus and Bacteria & $\begin{array}{l}\text { Antibacterial and } \\
\text { antifungal activity }\end{array}$ & [103] \\
\hline Metallic NPs & Microbes & $\begin{array}{l}\text { Antibacterial and } \\
\text { antifungal activity }\end{array}$ & [104] \\
\hline AgNPs & Fusarium culmorum & Antifungal activity & [105] \\
\hline
\end{tabular}


Table 2. Cont

\begin{tabular}{|c|c|c|c|}
\hline Nanoparticle & Pathogen & Effect & Reference \\
\hline Chitosan NPs & Streptococcus & Antibacterial activity & [106] \\
\hline AuNPs & Candida albicans & Antifungal activity & [107] \\
\hline AuNPs & Escherichia coli, Staphylococcus aureus & Antibacterial activity & [108] \\
\hline ZnO NPs & Ralstonia solanacearum & Antibacterial activity & [109] \\
\hline $\mathrm{ZnO}$ NPs & Botrytis, Escherichia & $\begin{array}{l}\text { Antibacterial and } \\
\text { antifungal activity }\end{array}$ & [110] \\
\hline $\mathrm{ZnO}$ NPs & Fusarium oxysporum, Aspergillus niger & $\begin{array}{l}\text { Antibacterial and } \\
\text { antifungal activity }\end{array}$ & [111] \\
\hline $\mathrm{ZnO}$ NPs & $\begin{array}{c}\text { Alternaria alternate, Fusarium oxysporum, Rhizopus } \\
\text { stolonifer and Mucor plumbeus }\end{array}$ & $\begin{array}{l}\text { Inhibit germination of } \\
\text { spores of fungi }\end{array}$ & [112] \\
\hline ZnO NPs & Botrytis cinerea and Penicillium expansum & Significantly inhibit growth & [113] \\
\hline ZnO NPs & Psedomanas sp. and Fusarium sp. & $\begin{array}{l}\text { Antibacterial and } \\
\text { antifungal activity }\end{array}$ & [114] \\
\hline $\mathrm{TiO}_{2}$ NPs & $\begin{array}{c}\text { Xanthomonas hortorum pv. pelargonii, X. axonopodis } \\
\text { pv. Poinsettiicola }\end{array}$ & Antibacterial activity & [115] \\
\hline
\end{tabular}

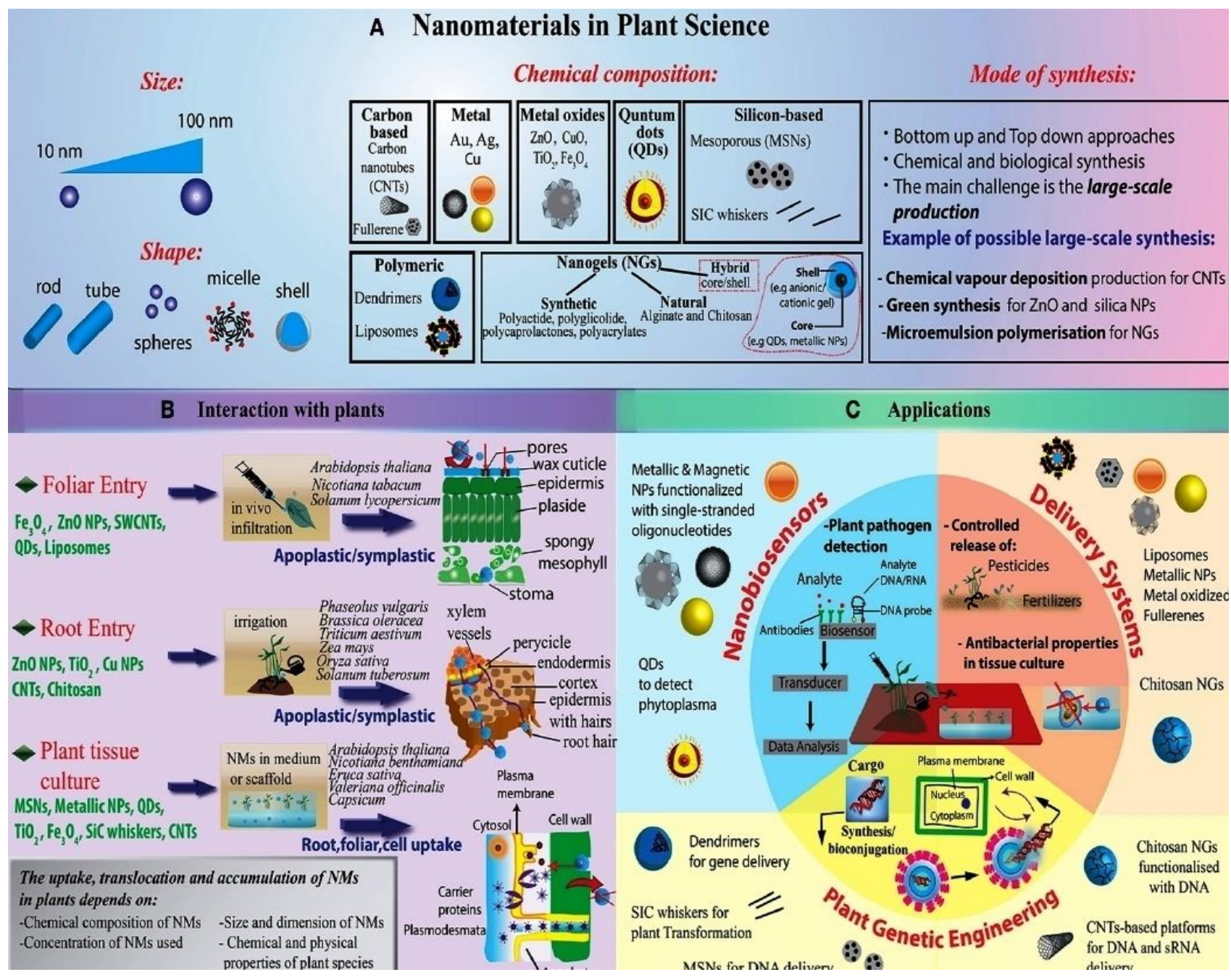

Figure 3. (A) Different types of nanoparticles. (B) Schematic presentation of delivery methods of different nanoparticles and translocation in plants. (C) Various applications of nanoparticles (Taken from Sanzari et al. [116]). 
The inhibitory action of nanoparticles on fungi and bacteria includes disruption by pore formation in the cell membrane, disturbance in membrane potential, cell wall damage, direct attachment to the cell surface, DNA damage, cell cycle arrest, the inhibition of enzyme activity and reactive oxygen species (ROS) generation, and this finally leads to death. Nanoparticles generate the ROS, which causes damage to the cellular structures. The different components of reactive oxygen species include free radicals, such as hydrogen peroxide $\left(\mathrm{H}_{2} \mathrm{O}_{2}\right)$, superoxide $\left(\mathrm{O}_{2}^{-}\right)$, singlet oxygen $\left({ }^{1} \mathrm{O}_{2}\right)$, carbon dioxide radical $\left(\mathrm{CO}_{2}{ }^{-}\right)$, hydroxyl $\left(\mathrm{HO}^{\circ}\right)$, hydroperoxyl $\left(\mathrm{HO}_{2}\right)$, carbonate $\left(\mathrm{CO}_{3}{ }^{-}\right)$, peroxyl $\left(\mathrm{RO}_{2}\right)$, and alkoxyl $(\mathrm{RO})$, and nonradicals, such as ozone $\left(\mathrm{O}_{3}\right)$, nitric oxide $(\mathrm{NO})$, hypobromous acid ( $\left.\mathrm{HOBr}\right)$, hypochlorous acid $(\mathrm{HOCl})$, hypochlorite $\left(\mathrm{OCl}^{-}\right)$, peroxy nitrite $\left(\mathrm{ONOO}^{-}\right)$, organic peroxides $(\mathrm{ROOH})$, peroxo monocarbonate $\left(\mathrm{HOOCO}_{2}{ }^{-}\right)$, peroxy nitrous acid $(\mathrm{ONOOH})$ and peroxy nitrate $\left(\mathrm{O}_{2} \mathrm{NOO}-\right)$, and these nanoparticles accumulate in the membrane of bacteria or fungi, which leads to change in the permeability of the cell membrane and disturbs the proton motive force (PMF).Oxidative stress due to the higher concentration leads to single- and double-strand breaks and nitrogen base and pentose sugar lesions [103,104].

\section{Toxic Effect of Nanoparticles}

Nanomaterials' effect on organisms is largely dependent on the dose, size, and shape, the types of NPs, concentration, and the duration of exposure to NPs and the plant/animal species $[117,118]$. Nanoparticles at optimum concentration augment the plant's growth, but high concentrations of nanoparticles could be toxic for plants. Kushwah and Patel [119] observed that the optimum concentration of nano $\mathrm{TiO}_{2}$ in the Vicia faba plant ranged from 5-50 mg/L. Other studies proved that $\mathrm{TiO}_{2}$ NPs may induce stress in plants such as tomato, cucumber and spinach at high concentration [120]. Silver nanoparticles cause chromosomal aberrations in Vicia faba [121]. Lopez-Moreno et al. [122] reported that $\mathrm{CeO}_{2}$ nanoparticles can induce DNA damage in soybean.

\section{Conclusions}

In summary, the literature shows that food demands will increase with time, and to fulfill the demand of people, the present agricultural practices are not sufficient and chemicals used in agriculture as pesticides have a severe toxic effect on the environment. Thus, we need to develop an alternative approach that has a less toxic effect on the environment and that could help in fulfilling food demands. According to estimates, around 192.8 Mt chemical fertilizers were used in 2016-2017 in the whole world. The use of toxic chemicals and pesticides causes environmental pollution, which affects fauna and flora. Pathogens and pests induce resistance against fungicides and pesticides. Hence, optimizing of the use of toxic chemical pesticides and fungicides is needed. Nanotechnology is flamboyant and has provided nanostructure materials as pesticide and fertilizer carriers. Nanomaterials can develop smart fertilizers as they can enhance nutrient availability and reduce environmental pollution [123]. Novel nanotechnology can be an alternative that can reduce crop diseases and enhance crop yield. Previous studies reported a significant positive effect of nanomaterials on crop plants. This novel technology can reduce the use of toxic chemicals and pesticides that contaminate soil, the environment, and groundwater. Further research is needed to develop this technology on a large scale (Figure 4). 


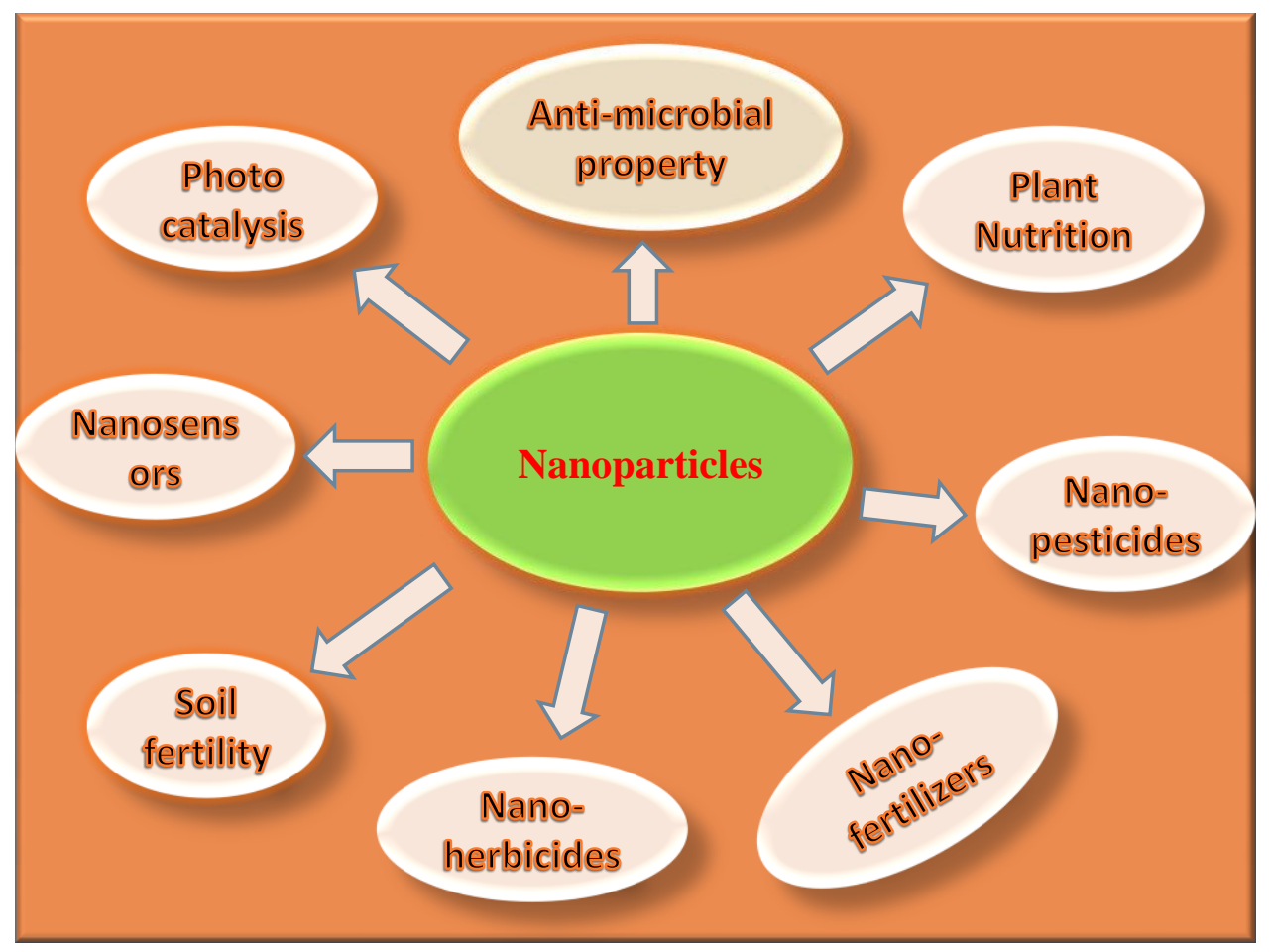

Figure 4. Diagram showing general applications of nanoparticles in agriculture.

Author Contributions: Writing—original draft: M.K. and A.U.K.; Writing—review \& editing: M.K., A.U.K., V.K.Y., K.K.Y. and M.M.C.P.; Conceptualization: G.K.S. and S.I. Data curation: M.A.H. and A.H.K.; Formal analysis: K.K.Y., N.M. and G.K.S.; Funding acquisition: M.A.H., M.M.C.P., A.H.K. and S.I.; Investigation: N.M., S.I. and G.K.S.; Methodology: M.K., A.U.K. and M.M.C.P.; Project administration: M.K., A.U.K., M.M.C.P.; Resources: M.A.H., K.K.Y., S.I., G.K.S. and A.H.K.; Software: K.K.Y., G.K.S. and N.M.; Supervision: A.U.K., M.M.C.P., A.H.K., V.K.Y., Validation: V.K.Y., M.A.H. and A.H.K.; Visualization: V.K.Y., S.I. and N.M. All authors have read and agreed to the published version of the manuscript.

Funding: Funding for this work has been provided by the Deanship of Scientific Research, KKU, Abha, Kingdom of Saudi Arabia, under research grant award number R.G.P2/85/41.

Institutional Review Board Statement: Not Applicable.

Informed Consent Statement: Not Applicable.

Data Availability Statement: The raw data used for this proposed work have been cited in the manuscript. Moreover, the derived data supporting the findings of this study have been graphically depicted and are available with the corresponding author on request.

Acknowledgments: The authors thankfully acknowledge the Deanship of Scientific Research, King Khalid University, Abha, for providing administrative and financial support. Funding for this work has been provided by the Deanship of Scientific Research, KKU, Abha, Kingdom of Saudi Arabia, under research grant award number R.G.P2/85/41. The authors also acknowledges the contribution and support provided by the University of Aveiro, Portugal. The authors wish to acknowledge the work of all the references used in this study.

Conflicts of Interest: The authors declare that there is no conflict of interest regarding the publication of this paper.

\section{References}

1. O'Neill, J.R. Irish Potato Famine; BDO: Edina, MN, USA, 2009; p. 1604535148.

2. Cabral-Pinto, M.M.S.; Inácio, M.; Neves, O.; Almeida, A.A.; Pinto, E.; Oliveiros, B.; Ferreira da Silva, E.A. Human health risk assessment due to agricultural activities and crop consumption in the surroundings of an industrial area. Expo. Health 2020, 12, 629-640. [CrossRef] 
3. F.A.O.; World Health Organization; WHO Expert Committee on Food Additives. Evaluation of Certain Contaminants in Food: EightyThird Report of the Joint FAO/WHO Expert Committee on Food Additives; World Health Organization: Geneve, Switzerland, 2017.

4. Tilman, D.; Knops, J.; Wedin, D.; Reich, P. Plant diversity and composition: Effects on productivity and nutrient dynamics of experimental grasslands. In Biodiversity and Ecosystem Functioning; Loreau, M., Naeem, S., Inchausti, P., Eds.; Oxford University Press: Oxford, UK, 2002; pp. 21-35.

5. Cohn, B.A.; Wolff, M.S.; Cirillo, P.M.; Sholtz, R.I. DDT and breast cancer in young women: New data on the significance of age at exposure. Environ. Health Perspect. 2007, 115, 1406-1414. [CrossRef] [PubMed]

6. Cabral-Pinto, M.M.S.; Marinho-Reis, P.; Almeida, A.; Pinto, E.; Neves, O.; Inácio, M.; Gerardo, B.; Freitas, S.; Simões, M.R.; Dinis, P.A.; et al. Links between cognitive status and trace element levels in hair for an environmentally exposed population: A case study in the surroundings of the Estarreja industrial area. Int. J. Environ. Res. Public Health 2019, 16, 4560. [CrossRef]

7. Khan, A.U.; Khan, M.; Khan, M.M. Antifungal and Antibacterial Assay by Silver Nanoparticles Synthesized from Aqueous Leaf Extract of Trigonella foenum-graecum. BioNanoScience 2019. [CrossRef]

8. Khan, M.; Khan, A.U.; Alam, M.J.; Park, S.; Alam, M. Biosynthesis of silver nanoparticles and its application against phytopathogenic bacterium and fungus. Int. J. Environ. Anal. Chem. 2019. [CrossRef]

9. Cabral-Pinto, M.M.S.; Silva, E.A.F.; Silva, M.M.V.G.; Melo-Gonçalves, P.; Candeias, C. Environmental risk assessment based on high-resolution spatial maps of potentially toxic elements sampled on stream sediments of Santiago, Cape Verde. Geosciences 2014, 4, 297-315. [CrossRef]

10. Cabral Pinto, M.M.S.; Marinho-Reis, P.; Almeida, A.; Freitas, S.; Simões, M.R.; Diniz, L.; Pinto, E.; Ramos, P.; Ferreira da Silva, E.; Moreira, P.I. Fingernail trace element content in environmentally exposed individuals and its influence on their cognitive status in ageing. Expo. Health 2019, 11, 181-194. [CrossRef]

11. BBC. BCC Research, Global Markets for Nanocomposites, Nanoparticles, Nanoclays, and Nanotubes. 2014. Available online: https: //www.bccresearch.com/market-research/nanotechnology/nanocomposites-market-nan021f.html?vsmaid=203/ (accessed on 19 October 2017).

12. Shang, Y.; Hasan, M.; Ahammed, G.J.; Li, M.; Yin, H.; Zhou, J. Applications of nanotechnology in plant growth and crop protection: A review. Molecules 2019, 24, 2558. [CrossRef]

13. Aslani, F.; Bagheri, S.; Muhd, J.N.; Juraimi, A.S.; Hashemi, F.S.; Baghdadi, A. Effects of engineered nanomaterials on plants growth: An overview. Sci. World J. 2014. [CrossRef] [PubMed]

14. Srivastav, A.; Yadav, K.K.; Yadav, S.; Gupta, N.; Singh, J.K.; Katiyar, R.; Kumar, V. Nano-Phytoremediation of Pollutants from Contaminated Soil Environment: Current Scenario and Future Prospects; Springer: Berlin/Heidelberg, Germany, 2018 ; pp. 383-401.

15. Chichiriccò, G.; Poma, A. Penetration and toxicity of nanomaterials in higher plants. Nanomaterials 2015, 5, 851-873. [CrossRef] [PubMed]

16. Eichert, T.; Kurtz, A.; Steiner, U.; Goldbach, H.E. Size exclusion limits and lateral heterogeneity of the stomatal foliar uptake pathway for aqueous solutes and water-suspended nanoparticles. Physiol. Plant 2008, 134, 151-160. [CrossRef]

17. Schreiber, L. Polar paths of diffusion across plant cuticles: New evidence for an old hypothesis. Ann. Bot. 2005, 95, 1069-1073. [CrossRef] [PubMed]

18. Zheng, L.; Hong, F.; Lu, S.; Liu, C. Effect of nano-TiO 2 on strength of naturally aged seeds and growth of spinach. Biol. Trace Elem. Res. 2005, 104, 83-91. [CrossRef]

19. Mahmoud, A.W.M.; Abdeldaym, E.A.; Abdelaziz, S.M.; El-Sawy, M.B.I.; Mottaleb, S.A. Synergetic Effects of Zinc, Boron, Silicon, and Zeolite Nanoparticles on Confer Tolerance in Potato Plants Subjected to Salinity. Agronomy 2020, 10, 19. [CrossRef]

20. Khan, M.; Siddiqui, Z.A. Zinc oxide nanoparticles for the management of Ralstonia solanacearum, Phomopsis vexans and Meloidogyne incognita incited disease complex of eggplant. Indian Phytopathol. 2018, 71, 355-364. [CrossRef]

21. Awasthi, A.; Bansal, S.; Jangir, L.K.; Awasthi, G.; Awasthi, K.K.; Awasthi, K. Effect of ZnO Nanoparticles on Germination of Triticum aestivum Seeds. Macromol. Symp. 2017, 376, 1700043. [CrossRef]

22. Rizwan, M.; Ali, S.; Rehman, M.Z. Effect of foliar applications of silicon and titanium dioxide nanoparticles on growth, oxidative stress, and cadmium accumulation by rice (Oryza sativa). Acta Physiol. Plant. 2019, 41, 35-42. [CrossRef]

23. Sabir, A.; Yazar, K.; Sabir, F.; Kara, Z.; Yazici, M.A.; Goksu, N. Vine growth, yield, berry quality attributes and leaf nutrient content of grapevines as influenced by seaweed extract (Ascophyllum nodosum) and nanosized fertilizer pulverizations. Sci. Hortic. 2014, 175, 1-8. [CrossRef]

24. Venkatachalam, P.; Jayaraj, M.; Manikandan, R.; Geetha, N.; Rene, E.R.; Sharma, N.C.; Sahi, S.V. Zinc oxide nanoparticles (ZnONPs) alleviate heavy metal-inducedtoxicity in Leucaena leucocephala seedlings: A physiochemicalanalysis. Plant Physiol. Biochem. 2017, 110, 59-69. [CrossRef]

25. Narendhran, S.; Rajiv, P.; Rajeshwari, S. Influence of zinc oxide nanoparticles on growth of Sesamum Indicum, L. in zinc deficient soil. Int. J. Pharm. Pharm. Sci. 2016, 8, 365-371.

26. Taheri, M.; Qarache, H.A.; Qarache, A.A.; Yoosefi, M. The effects of zinc-oxide nanoparticles on growth parameters of corn (SC704). STEM Fellowsh. J. 2015, 1, 17-20. [CrossRef]

27. Tarafdar, J.C.; Raliya, R.; Mahawar, H.; Rathore, I. Development of zinc nanofertilizer to enhance crop production in pearl millet (Pennisetum americanum). Agric.Res. 2014, 3, 257-262. [CrossRef]

28. Siddiqui, Z.A.; Khan, M.R.; Abdallah, E.F.; Parveen, A. Titanium dioxide and zinc oxide nanoparticles affect some bacterial diseases, and growth and physiological changes of beetroot. Int. J. Veg. Sci. 2019, 25, 409-430. [CrossRef] 
29. Raliya, R.; Biswas, P.; Tarafdar, J. $\mathrm{TiO}_{2}$ nanoparticle biosynthesis and its physiological effect on mung bean (Vigna radiata $\mathrm{L}$.). Biotechnol. Rep. 2015, 5, 22-26. [CrossRef]

30. Laware, S.L.; Raskar, S. Effect of titanium dioxide nanoparticles on hydrolytic and antioxidant enzymes during seed germination in onion. Int. J. Curr. Microbiol. Appl. Sci. 2014, 3, 749-760.

31. Rafique, R.; Arshad, M.; Khokhar, M.; Qazi, I.; Hamza, A.; Virk, N. Growth response of wheat to titania nanoparticles application. NUST J. Eng. Sci. 2014, 7, 42-46.

32. Mahmoodzadeh, H.; Nabavi, M.; Kashefi, H. Effect of Nanoscale Titanium Dioxide Particles on the Germination and Growth of Canola (Brassica napus). J. Ornam. Hortic. Plant 2013, 3, 25-32.

33. Qi, M.; Liu, Y.; Li, T. Nano-TiO($\left.{ }_{2}\right)$ improve the photosynthesis of tomato leaves under mild heat stress. Biol. Trace Elem. Res. 2013, 156, 323-328. [CrossRef] [PubMed]

34. Bao, S.L.; Chun, H.L.; Li, J.F.; Shu, C.Q.; Min, Y. Effect of TMS (nanostructured silicon dioxide) on growth of Changbai larch seedlings. J. For. Res. 2004, 15, 138-140.

35. Azimi, R.; Borzelabad, M.J.; Feizi, H.; Azimi, A. Interaction of $\mathrm{SiO}_{2}$ nanoparticles with seed prechilling on germination and early seedling growth of tall wheatgrass (Agropyron elongatum L.). Pol. J. Chem. Technol. 2014, 16, 25-29. [CrossRef]

36. Nair, R.; Varghese, S.H.; Nair, B.G.; Maekawa, T.; Yoshida, Y.; Kumar, D.S. Nanoparticulate material delivery to plants. Plant Sci. 2010, 179, 154-163. [CrossRef]

37. Siddiqui, M.H.; Al-Whaibi, M.H.; Faisal, M.; Al Sahli, A.A. Nano-silicon dioxide mitigates the adverse effects of salt stress on Cucurbita pepo L. Environ. Toxicol. Chem. 2014, 33, 2429-2437. [CrossRef]

38. Haghighi, M.; Afifipour, Z.; Mozafarian, M. The Effect of N-Si on Tomato Seed Germination under Salinity Levels. J. Biol. Environ. Sci. 2012, 6, 87-90.

39. Kasana, R.C.; Panwar, N.R.; Kaul, R.K.; Kumar, P. Biosynthesis and effects of copper nanoparticles on plants. Environ. Chem. Lett. 2017, 15, 233-240. [CrossRef]

40. Wang, Y.; Lin, Y.; Xu, Y.; Yin, Y.; Guo, H.; Du, W. Divergence in response of lettuce (var. ramosa Hort.) to copper oxide nanoparticles/microparticles as potential agricultural fertilizer. Environ. Pollut. Bioavailab. 2019, 31, 80-84. [CrossRef]

41. Zhao, L.J.; Peralta-Videa, J.R.; Rico, C.M.; Hernandez-Viezcas, J.A.; Sun, Y.; Niu, G.; Servin, A.; Nunez, J.E.; Duarte-Gardea, M.; Gardea-Torresdey, J.L. $\mathrm{CeO}_{2}$ and $\mathrm{ZnO}$ nanoparticles change the nutritional quality of cucumber (Cucumis sativus). J. Agric. Food Chem. 2014, 62, 2752-2759. [CrossRef]

42. Shinde, S.; Paralikar, P.; Ingle, A.P.; Rai, M. Promotion of seed germination and seedling growth of Zea mays by magnesium hydroxide nanoparticles synthesized by the filtrate from Aspergillus niger. Arab. J. Chem. 2020, 13, 3172-3182. [CrossRef]

43. Hussain, F.; Hadi, F.; Akbar, F. Magnesium oxide nanoparticles and thidiazuron enhance lead phyto accumulation and antioxidative response in Raphanus sativus L. Environ. Sci. Pollut. Res. 2019, 26, 30333-30347. [CrossRef]

44. Cai, L.; Liu, M.; Liu, Z.; Yang, H.; Sun, X.; Chen, J.; Ding, W. MgONPs can boost plant growth: Evidence from increased seedling growth, morpho-physiological activities, and Mg uptake in tobacco (Nicotiana tabacum L.). Molecules 2018, 23, 3375. [CrossRef] [PubMed]

45. Imada, K.; Sakai, S.; Kajihara, H.; Tanaka, S.; Ito, S. Magnesium oxide nanoparticles induce systemic resistance in tomato against bacterial wilt disease. Plant Pathol. 2016, 65, 551-560. [CrossRef]

46. Iqbal, M.; Raja, N.I.; Wattoo, F.H.; Hussain, M.; Ejaz, M.; Saira, H. Assessment of AgNPs exposure on physiological and biochemical changes and antioxidative defence system in wheat (Triticum aestivum L) under heat stress. IET NanobioTechnol. 2018, 16, 230-236. [CrossRef]

47. Mehta, C.M.; Srivastava, R.; Arora, S.; Sharma, A.K. Impact assessment of silver nanoparticles on plant growth and soil bacterial diversity. 3 Biotech 2016, 6, 254.

48. Pilon, L.; Spricigo, P.C.; Miranda, M.; Moura, M.R.; Assis, O.B.G.; Mattoso, L.H.C.; Ferreira, M.D. Chitosan nanoparticle coatings reduce microbial growth on fresh-cut apples while not afecting quality attributes. Int. J. Food Sci. Technol. 2015, 50, 440-448. [CrossRef]

49. Van, S.N.; Minh, H.D.; Anh, D.N. Study on chitosan nanoparticles on biophysical characteristics and growth of robusta cofee in green house. Biocatal. Agric. Biotechnol. 2013, 2, 289-294.

50. Das, C.K.; Srivastava, G.; Dubey, A.; Roy, M.; Jain, S.; Sethy, N.K.; Saxena, M.; Harke, S.; Sarkar, S.; Misra, K. Nano-iron pyrite seed dressing: A sustainable intervention to reduce fertilizer consumption in vegetable (beetroot, carrot), spice (fenugreek), fodder (alfalfa), and oilseed (mustard, sesamum) crops. Nanotechnol. Environ. Eng. 2016, 1, 2. [CrossRef]

51. Manikandan, A.; Sathiyabama, M. Preparation of chitosan nanoparticles and its effect on detached rice leaves infected with Pyricularia grisea. Int. J. Biol. Macromol. 2016, 1, 58-61. [CrossRef]

52. Hajirasouliha, M.; Jannesari, M.; Najafabadi, F.S.; Hashemi, M. Effect of novel chitosan nanoparticle coating on postharvest qualities of strawberry. In Proceedings of the 4th International Conference of Nanostructures, Kish Island, Iran, 12-14 March 2012.

53. Janmohammadi, M.; Sabaghnia, N. Effect of pre-sowing seed treatments with silicon nanoparticles on germinability of sunflower (Helianthus annuus). Botanica 2015, 21, 13-21. [CrossRef]

54. Roohizadeh, G.; Majd, A.; Arbabian, S. The effect of sodium silicate and silica nanoparticles on seed germination and growth in the Vicia faba L. Trop. Plant. Res. 2015, 2, 85-89. 
55. Kashyap, D.; Siddiqui, Z.A. Effect of silicon dioxide nanoparticles and Rhizobium leguminosarum alone and in combination on the growth and bacterial blight disease complex of pea caused by Meloidogyne incognita and Pseudomonas syringae pv. pisi. Arch. Phytopathol. Plant Prot. 2020, 3, 1-7. [CrossRef]

56. Adhikari, S.; Kundu, S.; Rao, S.A. Impact of $\mathrm{SiO}_{2}$ and Mo nanoparticles on seed germination of rice (Oryza sativa L.). Int. J. Agric. Food Sci. Tech. 2013, 4, 809-816.

57. Yinfeng, X.; Bo, L.; Gongsheng, T.A.O.; Qianqian, Z.; Chunxia, Z. Effects of nano-silicon dioxide on photosynthetic fluorescence characteristics of Indocalamus barbatus McClure. J. Nanjing For. Univer. 2012, 36, 59-63.

58. Suriyaprabha, R.; Karunakaran, G.; Yuvakkumar, R.; Rajendran, V.; Kannan, N. Silica nanoparticles for increased silica availability in maize (Zea mays. L) seeds under hydroponic conditions. Curr. Nanosci. 2012, 8, 902-908. [CrossRef]

59. Yuvakkumar, R.; Elango, V.; Rajendran, V.; Kannan, N.S.; Prabu, P. Influence of nanosilica powder on the growth of maize crop (Zea mays L.). Int. J. Green Nanotechnol. 2011, 3, 180-190. [CrossRef]

60. Lu, C.M.; Zhang, C.Y.; Wen, J.Q.; Wu, G.R.; Tao, M.X. Research on the effect of nanometer materials on germination and growth enhancement of Glycine max and its mechanism. Soybean Sci. 2002, 21, 68-172.

61. Zhao, L.; Ortiz, C.; Adeleye, A.S.; Hu, Q.; Zhou, H.; Huang, Y.; Keller, A.A. Metabolomics to detect response of lettuce (Lactuca sativa) to $\mathrm{Cu}(\mathrm{OH})_{2}$ nanopesticides: Oxidative stress response and detoxification mechanisms. Environ. Sci. Technol. 2016, 50, 9697-9707. [CrossRef] [PubMed]

62. Zhao, L.; Huang, Y.; Adeleye, A.S.; Keller, A.A. Metabolomics reveals $\mathrm{Cu}(\mathrm{OH})_{2}$ nanopesticide-activated anti-oxidative pathways and decreased beneficial antioxidants in spinach leaves. Environ. Sci. Technol. 2017, 51, 10184-10194. [CrossRef] [PubMed]

63. Yusefi-Tanha, E.; Fallah, S.; Rostamnejadi, A.; Pokhrel, L.R. Zinc oxide nanoparticles (ZnO NPs) as a novel nanofertilizer: Influence on seed yield and antioxidant defense system in soil grown soybean (Glycine max cv. Kowsar). Sci. Total Environ. 2020, 738, 140240. [CrossRef]

64. Singh, N.B.; Amist, N.; Yadav, K.; Singh, D.; Pandey, J.K.; Singh, S.C. Zinc oxide nanoparticles as fertilizer for the germination, growth and metabolism of vegetable crops. J. Nano Eng. Nano Manuf. 2013, 3, 1-12. [CrossRef]

65. Prasad, T.N.V.K.V.; Sudhakar, P.; Sreenivasulu, Y.; Latha, P.; Munaswamy, V.; Reddy, R.K.; Sreeprasad, T.S.; Sajanlal, P.R.; Pradeep, T. Effect of nanoscale zinc oxide particles on the germination, growth and yield of Peanut. J. Plant Nutr. 2012, 35, 905-927. [CrossRef]

66. Srivastava, G.; Das, C.K.; Das, A.; Singh, S.K.; Roy, M.; Kim, H.; Sethy, N.; Kumar, A.; Sharma, R.K.; Singh, S.K.; et al. Seed treatment with iron pyrite $\left(\mathrm{FeS}_{2}\right)$ nanoparticles increases the production of spinach. RSC Adv. 2014, 4, 58495-58504. [CrossRef]

67. Rezaei, F.; Moaveni, P.; Mozafari, H. Effect of different concentrations and time of nano $\mathrm{TiO}_{2}$ spraying on quantitative and qualitative yield of soybean (Glycine max L.) at Shahr-e-Qods. Iran. Biol. Forum. 2015, 7, 957-964.

68. Samadi, N.; Yahyaabadi, S.; Rezayatmand, Z. Effect of $\mathrm{TiO}_{2}$ and $\mathrm{TiO}_{2}$ Nanoparticle on Germination, Root and Shoot Length and Photosynthetic Pigments ofMentha Piperita. Int. J. Plant. Soil Sci. 2014, 3, 408-418. [CrossRef]

69. Reyhaneh, A.; Hassan, F.; Hosseini, M.K. Can Bulk and Nanosized Titanium Dioxide Particles Improve Seed Germination Features of Wheatgrass (Agropyron desertorum). Not. Sci. Biol. 2013, 5, 325-331.

70. Kim, S.W.; Jung, J.H.; Lamsal, K.; Kim, Y.S.; Min, J.S.; Lee, Y.S. Antifungal effects of silver nanoparticles (AgNPs) against various plant pathogenic fungi. Mycobiology 2012, 4, 53-58. [CrossRef]

71. Gautam, N.; Salaria, N.; Thakur, K.; Kukreja, S.; Yadav, N.; Yadav, R.; Goutam, U. Green silver nanoparticles for phytopathogen control. Proc. Natl. Acad. Sci. India Sect. B Biol. Sci. 2020, 90, 439-446. [CrossRef]

72. Rodríguez-Serrano, C.; Guzmán-Moreno, J.; Ángeles-Chávez, C.; Rodríguez-González, V.; Ortega-Sigala, J.J.; Ramírez-Santoyo, R.M.; Vidales-Rodríguez, L.E. Biosynthesis of silver nanoparticles by Fusarium scirpi and its potential as antimicrobial agent against uropathogenic Escherichia coli biofilms. PLoS ONE 2020, 15, e0230275. [CrossRef]

73. Hussein, E.A.M.; Al-Hajry, A.M.; Harraz, F.A.; Ahsan, M.F. Biologically synthesized silver nanoparticles for enhancing tetracycline activity against Staphylococcus aureus and Klebsiella pneumonia. Braz. Arch. Biol. Technol. 2019, 62, 25. [CrossRef]

74. Shehzad, A.; Qureshi, M.; Jabeen, S.; Ahmad, R.; Alabdalall, A.H.; Aljafary, M.A.; Al-Suhaimi, E. Synthesis, Characterization and antibacterial activity of silver nanoparticles using Rhazya stricta. Peer J. 2018, 6, e6086. [CrossRef]

75. Mohanta, Y.K.; Panda, S.K.; Bastia, A.K.; Mohanta, T.K. Biosynthesis of silver nanoparticles from protium serratum and Potential Impacts on food safety and control. Front. Microbiol. 2017. [CrossRef] [PubMed]

76. Abdelmalek, G.A.M.; Salaheldin, T.A. Silver nanoparticles as a potent fungicide for citrus phytopathogenic fungi. J. Nanomed. Res. 2016, 3, 1-8.

77. Krishnaraj, C.; Ramachandran, R.; Mohan, K.; Kalaichelvan, P. Optimization for rapid synthesis of silver nanoparticles and its effect on phytopathogenic fungi. Spectrochim. Acta A 2012, 93, 95-99. [CrossRef] [PubMed]

78. Jo, Y.-K.; Kim, B.H.; Jung, G. Antifungal activity of silver ions and nanoparticles on phytopathogenic fungi. Plant. Dis. 2009, 93, 1037-1043. [CrossRef] [PubMed]

79. Shahryari, F.; Rabiei, Z.; Sadighian, S. Antibacterial activity of synthesized silver nanoparticles by sumac aqueous extract and silver-chitosan nanocomposite against Pseudomonas syringae pv. syringae. J. Plant. Pathol. 2020, 102, 469-475. [CrossRef]

80. Divya, K.; Vijayan, S.; George, T.K.; Jisha, M. Antimicrobial properties of chitosan nanoparticles: Mode of action and factors affecting activity. Fibers Polym. 2017, 18, 221-230. [CrossRef]

81. Xing, K.; Shen, X.; Zhu, X.; Ju, X.; Miao, X.; Tian, J.; Feng, Z.; Peng, X.; Jiang, J.; Qin, S. Synthesis and in vitro antifungal efcacy of oleoyl-chitosan nanoparticles against plant pathogenic fungi. Int. J. Biol. Macromol. 2016, 82, 830-836. [CrossRef] 
82. Dang, H.; Fawcett, D.G.; Poinern, J. Green synthesis of gold nanoparticles from waste macadamia nuts shells and their antimicrobial activity against Escherichia coli and Staphylococcus epidermis. Int. J. Res. Med. Sci. 2019. [CrossRef]

83. Attar, A.; Yapaoz, M.A. Biomimetic synthesis, characterization and antibacterial efficacy of ZnO and Au nanoparticles using Echinacea flower extract precursor. Mater. Res. Express. 2018, 5, 5. [CrossRef]

84. Jayaseelan, C.; Ramkumar, R.; Rahuman, A.A.; Perumal, P. Green synthesis of gold nanoparticles using seed aqueous extract of Abelmoschus esculentus and its antifungal activity. Ind. Crops Prod. 2013, 45, 423-429. [CrossRef]

85. Fan, Q.; Liao, Y.Y.; Kunwar, S.; Da Silva, S.; Young, M.; Santra, S.; Paret, M.L. Antibacterial effect of copper composites against Xanthomonas euvesicatoria. Crop Prot. 2020, 139, 105366. [CrossRef]

86. Huang, S.; Wang, L.; Liu, L.; Hou, Y.; Li, L. Nanotechnology in agriculture, livestock, and aquaculture in China. A review. Agron. Sustain. Dev. 2015, 35, 369-400. [CrossRef]

87. Giannousi, K.; Avramidis, I.; Dendrinou-Samara, C. Synthesis, characterization and evaluation of copper based nanoparticles as agrochemicals against Phytophthora infestans. RSC Adv. 2013, 3, 21743-21752. [CrossRef]

88. Sharma, N.; Jandaik, S.; Kumar, S.; Chitkara, M.; Sandhu, I.S. Synthesis, characterisation and antimicrobial activity of manganeseand iron-doped zinc oxide nanoparticles. J. Exp. Nanosci. 2015, 11, 54-71. [CrossRef]

89. Derbalah, A.; Shenashen, M.; Hamza, A.; Mohamed, A.; El Safty, S. Antifungal activity of fabricated mesoporous silica nanoparticles against early blight of tomato. Egypt. J. Basic Appl. Sci. 2018, 5, 145-150. [CrossRef]

90. Akpinar, I.; Sar, T.; Unal, M. Antifungal effects of silicon dioxide nanoparticles $\left(\mathrm{SiO}_{2} \mathrm{NPs}\right)$ against various plant pathogenic fungi. In International Workshop: Plant Health: Challenges and Solutions; Tar, M., Baştaş, K.K., Eds.; Frontiers Media SA: Antalya, Turkey, 2017; ISBN 978-2-88945-218-7.

91. Park, H.J.; Kim, S.H.; Kim, H.J.; Choi, S.H. A new composition of nano sized silica-silver for control of various plant diseases. Plant Pathol. 2006, 22, 295-302. [CrossRef]

92. Jamdagni, P.; Khatri, P.; Rana, J.S. Green synthesis of zinc oxide nanoparticles using flower extract of Nyctanthesarbor-tristis and their antifungal activity. J. King Saud Uni. Sci. 2016. [CrossRef]

93. Navale, G.R.; Thripuranthaka, M.; Late, D.J.; Shinde, S.S. Antimicrobial Activity of ZnO Nanoparticles against Pathogenic Bacteria and Fungi. JSM Nanotechnol. Nanomed. 2015, 3, 10-33.

94. Rajiv, P.; Rajeshwari, S.; Venckatesh, R. Bio-Fabrication of zinc oxide nanoparticles using leaf extract of Parthenium hysterophorus L. and its size-dependent antifungal activity against plant fungal pathogens. Spectrochim. Acta A 2013, 112, 384-387. [CrossRef] [PubMed]

95. Gunalan, S.; Sivaraj, R.; Rajendran, V. Green synthesized ZnO nanoparticles against bacterial and fungal pathogens. Prog. Nat. Sci. Mater. 2012, 22, 693-700. [CrossRef]

96. Dimkpa, C.O.; McLean, J.E.; Britt, D.W.; Anderson, A.J. Antifungal activity of ZnO nanoparticles and their interactive effect with a biocontrol bacterium on growth antagonism of the plant pathogen Fusarium graminearum. Biometals 2013, 26, 913-924. [CrossRef] [PubMed]

97. Jayaseelan, C.; Rahuman, A.A.; Kirthi, A.V.; Marimuthu, S.; Santhoshkumar, T.; Bagavan, A.; Rao, K.V.B. Novel microbial route to synthesize $\mathrm{ZnO}$ nanoparticles using Aeromonas hydrophila and their activity against pathogenic bacteria and fungi. Spectrochim. Acta Part A Mol. Biomol. Spectrosc. 2012, 90, 78-84. [CrossRef]

98. Sar, T.; Akpinar, I.; Unal, M. Inhibitory effect of antifungal activity of Titanium Dioxide $\left(\mathrm{TiO}_{2}\right)$ nanoparticles on some pathogenic Fusarium isolates. In Proceedings of the International Workshop Plant Health: Challenges and Solutions, Antalya, Turkey, 23-28 April 2017. 78p.

99. Hamza, A.; El-Mogazy, S.; Derbalah, A. Fenton reagent and titanium dioxide nanoparticles as antifungal agents to control leaf spot of sugar beet under field conditions. J. Plant Prot. Res. 2016, 56, 270-278. [CrossRef]

100. Ardakani, A.S. Toxicity of silver, titanium and silicon nanoparticles on the root-knot nematode, Meloidogyne incognita, and growth parameters of tomato. Nematology 2013, 1-7. [CrossRef]

101. Kasemets, K.; Ivask, A.; Dubourgier, H.C.; Kahru, A. Toxicity of nanoparticles of ZnO, CuO and $\mathrm{TiO}_{2}$ to yeast Saccharomyces cerevisiae. Toxicol. Vitr. 2009, 23, 1116-1122. [CrossRef] [PubMed]

102. Cui, H.; Zhang, P.; Gu, W.; Jiang, J. Application of anatasa $\mathrm{TiO}_{2}$ sol derived from peroxotitannic acid in crop diseases control and growth regulation. NSTI Nanotech. 2009, 2, 286-289.

103. Slavin, Y.N.; Asnis, J.; Häfeli, U.O.; Bach, H. Metal nanoparticles: Understanding the mechanisms behind antibacterial activity. J. NanobioTechnol. 2017, 15, 65. [CrossRef]

104. Singh, J.; Vishwakarma, K.; Ramawat, N.; Rai, P.; Singh, V.K.; Mishra, R.K.; Kumar, V.; Tripathi, D.K.; Sharma, S. Nanomaterials and microbes' interactions: A contemporary overview. 3 Biotech 2019, 9, 1-4. [CrossRef]

105. Kasprowicz, M.J.; Kozioł, M.; Gorczyca, A. The effect of silver nanoparticles on phytopathogenic spores of Fusarium culmorum. Can. J. Microbiol. 2010, 56, 247-253. [CrossRef] [PubMed]

106. De Paz, L.E.; Resin, A.; Howard, K.A.; Sutherland, D.S.; Wejse, P.L. Antimicrobial effect of chitosan nanoparticles on Streptococcus mutans biofilms. Appl. Environ. Microbiol. 2011, 77, 3892-3895. [CrossRef]

107. Aljabali, A.A.; Akkam, Y.; Al Zoubi, M.S.; Al-Batayneh, K.M.; Al-Trad, B.; Abo Alrob, O.; Alkilany, A.M.; Benamara, M.; Evans, D.J. Synthesis of gold nanoparticles using leaf extract of Ziziphus zizyphus and their antimicrobial activity. Nanomaterials 2018, 3, 174. [CrossRef] 
108. Yuan, C.G.; Huo, C.; Gui, B.; Cao, W.P. Green synthesis of gold nanoparticles using Citrus maxima peel extract and their catalytic/antibacterial activities. IET NanobioTechnol. 2016, 11, 523-530. [CrossRef]

109. Khan, R.A.; Tang, Y.; Naz, I.; Alam, S.S.; Wang, W.; Ahmad, M.; Najeeb, S.; Ali, A.; Rao, C.; Li, Y.; et al. Management of Ralstonia solanacearum in tomato using ZnO nanoparticles synthesized through Matricaria chamomilla. Plant Disease. 2021, 28. [CrossRef]

110. Kairyte, K.; Kadys, A.; Luksiene, Z. Antibacterial and antifungal activity of photoactivated ZnO nanoparticles in suspension. J. Photochem. Photobiol. 2013, 128, 78-84. [CrossRef]

111. Patra, P.; Mitra, S.; Debnath, N.; Goswami, N. Biochemical-Biophysical-and microarray-based antifungal evaluation of the buffer-mediated synthesized nano zinc oxide: An in vivo and in vitro toxicity study. Langmuir 2012, 28, 16966-16978. [CrossRef] [PubMed]

112. Wani, A.H.; Shah, M.A. unique and profound effect of $\mathrm{MgO}$ and $\mathrm{ZnO}$ nanoparticles on some plant pathogenic fungi. J. Appl. Pharm. Sci. 2012, 2, 40-44.

113. He, L.; Liu, Y.; Mustapha, A.; Lin, M. Antifungal activity of zinc oxide nanoparticles against Botrytis cinerea and Penicillium expansum. Microbiol. Res. 2011, 166, 207-215. [CrossRef] [PubMed]

114. Sharma, D.; Rajput, J.; Kaith, B.S.; Kaur, M.; Sharma, S. Synthesis of ZnO nanoparticles and study of their antibacterial and antifungal properties. Thin Solid Films 2010, 519, 1224-1229. [CrossRef]

115. Norman, D.J.; Chen, J. Effect of foliar application of titanium dioxide on bacterial blight of geranium and Xanthomonas leaf spot of poinsettia. Hortic. Sci. 2011, 46, 426-428. [CrossRef]

116. Sanzari, I.; Leone, A.; Ambrosone, A. Nanotechnology in Plant Science: To Make a Long Story Short. Front. Bioeng. Biotechnol. 2019, 7, 120. [CrossRef]

117. Naushad, M. Surfactant assisted nano-composite cation exchanger: Development, characterization and applications for the removal of toxic $\mathrm{Pb}^{2+}$ from aqueous medium. Chem. Eng. J. 2014, 235, 100-108. [CrossRef]

118. Albadarin, A.B.; Collins, M.N.; Naushad, M.; Shirazian, S.; Walker, G.; Mangwandi, C. Activated lignin-chitosan extruded blends for efficient adsorption of methylene blue. Chem. Eng. J. 2017, 307, 264-272. [CrossRef]

119. Kushwah, K.S.; Patel, S. Effect of Titanium Dioxide Nanoparticles ( $\mathrm{TiO}_{2} \mathrm{NPs}$ ) on Faba bean (Vicia faba L.) and Induced Asynaptic Mutation: A Meiotic Study. J. Plant. Growth Regul. 2020, 39, 1107-1118. [CrossRef]

120. Gohari, G.; Mohammadi, A.; Akbari, A.; Panahirad, S.; Dadpour, M.R.; Fotopoulos, V.; Kimura, S. Titanium dioxide nanoparticles $\left(\mathrm{TiO}_{2} \mathrm{NPs}\right)$ promote growth and ameliorate salinity stress effects on essential oil profile and biochemical attributes of Dracocephalum moldavica. Sci. Rep. 2020, 10, 912. [CrossRef]

121. Patlolla, A.K.; Berry, A.; May, L.; Tchounwou, P.B. Genotoxicity of Ag NPs in Vicia faba: A pilot study on the environmental monitoring of NPs. Int. J.Environ.Res. Public Health 2012, 9, 1649-1662. [CrossRef] [PubMed]

122. Lopez-Moreno, M.L.; de la Rosa, G.; Hernández-Viezcas, J.; Castillo-Michel, H.; Botez, C.E.; Peralta-Videa, J.R.; Gardea-Torresdey,

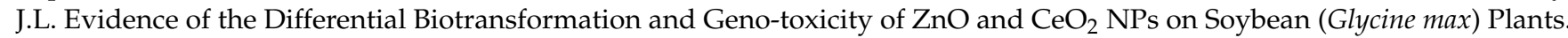
Environ. Sci. Technol. 2010, 44, 7315-7320. [CrossRef] [PubMed]

123. Manjunatha, S.B.; Biradar, D.P.; Aladakatti, Y.R. Nanotechnology and its applications in agriculture: A review. J. Farm Sci. 2016, $29,1-3$ 